\title{
Histopathological Spectrum of Non-Malignant Lesions of Gastrointestinal Tract- An Institutional Study.
}

\author{
Wasim M. Khatib ${ }^{1}$, Rakesh B. Demde ${ }^{2}$, Vidya C. Aher ${ }^{2}$, Pankti M.Patel. ${ }^{2}$ \\ ${ }^{1}$ Assistant Professor, Department of Pathology, Krishna Institute of Medical Sciences, Karad, \\ Maharashtra, India. \\ ${ }^{2}$ Assistant Lecturer, Department of Pathology, Krishna Institute of Medical Sciences, Karad, \\ Maharashtra, India.
}

\begin{abstract}
:
Background: Gastrointestinal tract (GIT) diseases are one of the most common entities we come across in dayto-day practice. Given its sheer length and extent, there is a possibility of various pathologies affecting individual segments of the entire tract. Ranging from congenital anomalies, acute and/or chronic inflammations, inflammatory conditions to neoplastic conditions including both, benign as well as malignant lesions, the spectrum of diseases which can occur individually or in combination with various segments is wide. In the present study, we aim to understand and analyse various diseases encountered during the study period. Materials and Method: The present study was conducted in the department of Pathology, of a tertiary rural care hospital, over a period of 1 year (Jan 2014 to Dec 2014). All non-malignant cases which were confirmed on histopathology were studied. Detailed clinical and radiological history was obtained. All the data was then further analysed.

Result: A total of 263 cases were received. Of these, 198 (75.29\%) cases were non-malignant, whereas 65 (24.71\%) cases were malignant. 100 (50.50\%) were males while 98 (49.50\%) were females. Mean age was 48.2 years. Most common affected site was appendix with $146(73.73 \%)$ cases. Chronic inflammatory lesions were most commonly encountered with 62 (31.31\%) cases. We had 02 (1.01\%) inflammatory bowel diseases (IBD) and $03(1.52 \%)$ polyps.

Conclusion: Numerous infectious, inflammatory and other conditions were encountered in our study. A plethora of lesions can potentially affect the GI tract. Accurate diagnosis of these conditions is of paramount importance.
\end{abstract}

Keyword: Gastrointestinal tract, Non-malignant, IBD.

\section{Introduction}

Gastrointestinal tract (GI tract) is a hollow organ extending from the oral cavity, encompassing the oesophagus, stomach, small intestine, large intestine and anus. The various lesions that can affect the GI tract can be classified as congenital anomalies, infectious, inflammatory, polyps, hamartomatous conditions, benign and malignant neoplasms. Majority of the cases received for evaluation are generally endoscopic biopsies, however, resected specimens are received for histopathological examination. Endoscopy, however has the advantage of being used both diagnostically and therapeutically. ${ }^{[1]}$ It is a simple and well accepted procedure which helps in early diagnosis and provision for better treatment options.

Within the upper GI tract, the oesophagus and stomach are the most favoured sites for bacterial and fungal infections, vascular disorders, caustic injuries and neoplasms. ${ }^{[2]}$ Similarly, the lower GI tract is also a site for various infectious, inflammatory, idiopathic and polypoidal conditions along with neoplasms. ${ }^{[3,4]}$ Hirschsprung disease, acute and/ or chronic inflammatory conditions, benign or malignant polyps, inflammatory bowel diseases and benign and malignant neoplasms are among the few conditions which can be encountered in the lower GI tract predominantly. ${ }^{[5]}$ Septicemia, ulceration, perforation peritonitis are few complications that can arise throughout the GI tract.

Biopsy is the gold standard in the diagnosis of any lesion. In our study, we received both endoscopic biopsies as well as resected specimens. Herein, we have made an attempt to analyze the various lesions encountered in our study under clinical and radiological guidance with the final diagnosis resting on histopathological examination.

\section{Materials and Method}

The present study was conducted in the department of Pathology of a tertiary rural care hospital, over a period of 1 year (Jan 2014 to Dec 2014). All clinically diagnosed non-malignant cases which were confirmed on histopathology were studied. Detailed clinical and radiological history was obtained to aid the diagnosis. All the data was then further analysed. 
The present work is a descriptive, cross sectional and analytical type of study. All endoscopic biopsies, resected specimens and slides for review were studied. The lesions from the oral cavity (excluding the nasopharynx), oesophagus, stomach, duodenum, ileum, caecum, all the parts of colon, rectum and anal canal were included in the analysis.

The resected specimens were fixed according to standard protocols. Appropriate and adequate sections were taken so as to cover the entire specimen. Lymph nodes whereever possible were resected. All endoscopic biopsies were submitted directly for processing. All the sections were processed under standard guidelines and were stained with hematoxylin and eosin stain and studied under light microscopy. The demographic details, clinical and radiological investigations were obtained from the requisition form, treating physicians and patient file. All the data were statistically analyzed and studied. All malignant lesions were excluded from this study.

\section{Results}

A total of 263 cases were received in the one year period. Of these, 198 cases $(75.29 \%)$ were nonmalignant whereas, $65(24.71 \%)$ cases were malignant. The appendix was the most commonly encountered organ with $146(73.73 \%)$ cases followed by the large intestine with $20(10.10 \%)$ cases. In the large intestine, most frequently affected was colon excluding the sigmoid colon with 06 cases followed by the anal canal with 05 cases. Caecum was affected in 04 cases. (Table 1)

Table 1 -Distribution of lesions according to site

\begin{tabular}{|c|c|c|}
\hline Site & Number of cases & Percentage (\%) \\
\hline Oral cavity & 11 & 5.56 \\
\hline Oesophagus & 07 & 3.54 \\
\hline Stomach & 02 & 1.01 \\
\hline Appendix & 146 & 73.73 \\
\hline Small Intestine & 12 & 6.06 \\
\hline Large Intestine & 20 & 10.10 \\
\hline Total & 198 & 100 \\
\hline
\end{tabular}

Duodenum was affected in 02 patients whereas ileal lesions were encountered in 06 cases of the 12 $(6.06 \%)$ cases affecting the small intestine. 02 cases $(1.01 \%)$ of gastritis were noted. There was a single case of Candidial oesophagitis. In our study, upper oesophagus was affected more frequently. (Table 1) (Table 1)

The oral cavity was affected in 11 cases $(5.56 \%)$ of the 198 . Tongue was affected in $04(2.02 \%)$ cases.

Considering all the sites, 21-40 years age group was the most commonly affected with 90 (44.46\%) cases followed by less than 20 years of age group with $44(22.22 \%)$ cases. (Table 2) There was an equivocal distribution of cases in the 41-70 years of age group at all sites. The youngest patient was an eight day female operated for failure to pass stools whereas the oldest patient was an 85 year old male operated for frequent change in bowel habits, fever and obstruction. The mean age of oral lesions was 59.6 years which was the oldest when compared with the rest of the GI tract. Small intestinal lesions were more frequently encountered in the middle age group with the mean age affected being 39.1 years. Appendiceal lesions were more frequently encountered in the younger age group with mean age affected being 22.9 years. (Table 2)

Table 2 - Distribution of lesions according to age

\begin{tabular}{|c|c|c|c|c|c|c|}
\hline Age in years & Oral & Oesophagus & Stomach & Appendix & $\begin{array}{c}\text { Small } \\
\text { Intestine }\end{array}$ & $\begin{array}{c}\text { Large } \\
\text { Intestine }\end{array}$ \\
\hline$<20$ & 02 & - & - & 38 & 02 & 02 \\
\hline $21-30$ & - & - & - & 42 & 02 & 03 \\
\hline $31-40$ & - & - & - & 37 & 02 & 04 \\
\hline $41-50$ & 01 & 03 & 01 & 12 & 03 & 03 \\
\hline $51-60$ & 03 & 02 & - & 10 & 01 & 04 \\
\hline $61-70$ & 03 & - & 01 & 05 & 01 & 04 \\
\hline $71-80$ & 01 & 01 & - & 02 & - & - \\
\hline$>80$ & 01 & - & - & - & 01 & - \\
\hline Total & 11 & 07 & 02 & 146 & 12 & 20 \\
\hline
\end{tabular}

There was a slight male preponderance noted in our study with $100(50.50 \%)$ cases. $98(49.50 \%)$ cases were seen in females. Among both the sexes, apart from appendix, most frequently affected region was large intestine followed by oral cavity with $20(10.10 \%)$ and $11(5.56 \%)$ cases respectively. Male to female ratio of 1.02:1 was noted. (Table 3)

Table 3 Distribution of lesions according to other demographic details

\begin{tabular}{|c|c|c|c|c|c|c|}
\hline Parameter & Oral cavity & Oesophagus & Stomach & Appendix & $\begin{array}{c}\text { Small } \\
\text { Intestine }\end{array}$ & $\begin{array}{c}\text { Large } \\
\text { Intestine }\end{array}$ \\
\hline
\end{tabular}


Histopathological Spectrum Of Non-Malignant Lesions Of Gastrointestinal Tract- A.....

\begin{tabular}{|l|c|c|c|c|c|c|}
\hline Male & 06 & 05 & 00 & 71 & 07 & 11 \\
\hline Female & 05 & 02 & 02 & 75 & 05 & 09 \\
\hline M:F & 59.6 & 58.7 & 57.5 & 22.9 & 39.1 & 51.7 \\
\hline $\begin{array}{l}\text { Mean Age } \\
\text { (years) }\end{array}$ & & $1.02: 1$ \\
\hline
\end{tabular}

When segregated, we came across numerous diagnoses affecting individual segments of the GI tract. However, we came across many nonspecific inflammatory lesions where pin point diagnosis to particular etiological entity could not be made. When dealing with the oral cavity, majority of the lesions were epithelial proliferative in nature with $05(2.52 \%)$ cases. Pseudoepitheliomatous hyperplasia, acanthosis were diagnoses put forth which correlated with the clinical diagnosis of leukoplakia. The tongue was affected in $04(2.02 \%)$ cases. All four were vascular masses which were diagnosed as lobular capillary hemangioma.

We received oesophageal endoscopic biopsies which were mostly acute inflammatory and/or necrotic in nature. There was a single case of a 50 year old immunocompromised male whose oesophageal scraping revealed Candidal infection.

Among appendiceal lesions, chronic followed by acute on chronic appendicitis were the frequently encountered diagnoses with $59(29.80 \%)$ and $47(23.74 \%)$ cases respectively. 02 cases of eosinophilic appendicitis and a single case of granulomatous inflammation in the appendix were observed in our study.

Table 4 - Distribution of lesions according to histopathological diagnosis

\begin{tabular}{|c|c|c|c|c|c|c|}
\hline Diagnosis & Oral & $\begin{array}{c}\text { Oesophagu } \\
\text { s }\end{array}$ & Stomach & Appendix & Small Intestine & Large Intestine \\
\hline $\begin{array}{c}\text { Acute } \\
\text { inflammation }\end{array}$ & 02 & 03 & - & 30 & 02 & 02 \\
\hline $\begin{array}{c}\text { Chronic } \\
\text { inflammation }\end{array}$ & - & - & 01 & 59 & - & 02 \\
\hline $\begin{array}{c}\text { Acute on } \\
\text { chronic } \\
\text { inflammation }\end{array}$ & - & - & 01 & 47 & 02 & 04 \\
\hline $\begin{array}{c}\text { Necrosis/ } \\
\text { gangrene }\end{array}$ & - & 03 & - & 06 & 03 & 05 \\
\hline Perforation & - & - & - & 02 & 02 & 01 \\
\hline IBD & - & - & - & - & 02 & - \\
\hline Polyps & - & - & - & - & - & 03 \\
\hline Obstruction & - & - & - & - & 01 & 01 \\
\hline $\begin{array}{c}\text { Granulomatous } \\
\text { Inflammation }\end{array}$ & - & - & - & 01 & - & - \\
\hline $\begin{array}{c}\text { Eosinophilic } \\
\text { inflammation }\end{array}$ & - & - & - & 02 & - & - \\
\hline Vascular lesion & 04 & - & - & - & - & - \\
\hline $\begin{array}{c}\text { Epithelial } \\
\text { proliferation }\end{array}$ & 05 & - & - & - & - & 20 \\
\hline Other & - & 01 & - & - & - & 12 \\
\hline Total & 11 & 07 & 02 & 146 & & - \\
\hline
\end{tabular}

(*IBD= Inflammatory bowel disease)

Obstructive symptoms were noted in $03(1.52 \%)$ cases. An eight day female baby was operated for intussusception. We encountered 03 polyps. Hyperplastic polyp was diagnosed in a 55 years old male who presented with bleeding per rectum, Juvenile polyp was diagnosed in a 05 years old boy and a hamartomatous polyp was noted in a 23 years old female. (Table 4 )

We had $02(1.01 \%)$ cases of inflammatory bowel disease, both of which were seen in the ileum. One was diagnosed as Ulcerative colitis in a 51 years old female whereas the other was diagnosed as indeterminate colitis which was observed in a 47 years old male. (Table 4)

\section{Discussion}

When dealing with gastrointestinal tract, the upper GI limited upto the stomach and the lower GI extending from duodenum upto the anal canal, show different results when studied for age affected, gender affected and lesions encountered. In our study, there was an equal incidence of males and females affected in the lower GI tract whereas a slight male preponderance was noted in the upper GI tract. Similar findings were noted by Shennk MM et al.[6] In our study, 41-60 years age group was the most commonly affected which also was in concordance with the above mentioned study. ${ }^{[6]}$

In the lower GI tract, 21 to 51 years age group was most commonly affected. Our findings were in concordance with the studies done by Hassan Abdulla Al- aquili et al who showed most common age group affected to be 21-60 years. ${ }^{[7]}$ In our study, we had 03 endoscopically removed polypectomy specimen which 
were diagnosed as juvenile, hyperplastic and hamartomatous polyp each. Though there was no preponderant polypoidal diagnosis, the fact that the large intestine showed maximum polyps is in concordance with other studies which state that neoplastic as well as non-neoplastic polyps are more commonly encountered in the large intestine ${ }^{[8]}$ The number of polypectomy specimen is less as seen in our study. This points towards a need for better implementation of colonoscopic and upper GI endoscopic surveillance programmes.

Inflammatory bowel disease particularly Ulcerative colitis have a notoriety to culminate into malignancy. Amongst this, ulcerative colitis has the highest predisposition. ${ }^{[9]}$ Patients presenting with pain in abdomen, vomiting, diarrhoea with or without constipation and non responding to the treatment can be categorized as IBD. Routine colonoscopic surveillance or targeted colonoscopy is of prime importance when dealing with such cases. In proved cases, any sign of dysplasia especially high grade dysplasia, advocates colectomy as the next immediate intervention. ${ }^{[10]}$

Gastritis can be classified into chronic superficial, chronic nonspecific, erosive gastritis, gastritis with intestinal metaplasia and $\mathrm{H}$. pylori positive gastritis. We came across $02(1.01 \%)$ cases of chronic nonspecific gastritis. Cultures of both of these cases came back negative for H. pylori. Both the cases were treated accordingly.

The duodenum has a rapidly regenerative epithelial lining which can be easily affected by trauma, toxins and similar insults. ${ }^{[10]}$ We had only 02 cases of acute and acute on chronic inflammation of the duodenum. Neil A Shepherd et al, showed considerable non-neoplastic lesions affecting the duodenum. ${ }^{[1]]}$ Granulomatous inflammation especially when seen in the intestine particularly large intestine should be investigated first for tuberculosis in a country like India. Crohn disease is a close mimic to tubercular enteritis and hence IBD should be considered in the differential of granulomatous enteritis. Microscopically, subtle changes such as absence of surrounding lymphocytes and caseation which are seen in granuloma of IBD especially Crohn's should be kept in mind.

The treatment protocol for IBD is completely different from tuberculosis and other infections. Polypectomy and for that matter routine endoscopic surveillance is highly recommended for any new growths within the GI tract. Acute and/or chronic inflammations should be treated accordingly. Epithelial proliferative conditions such as pseudoepithelomatous hyperplasia warrant follow up.

\section{Conclusion}

A wide range of lesions have a potential to occur anywhere in the GI tract. From trivial inflammations which warrant minimal therapeutic intervention to inflammatory conditions such as Ulcerative colitis and Crohn disease which require serious therapeutic interventions, the range of non-malignant lesions that can occur, has a vast differential. Accurate diagnosis of individual entities either on endoscopy or gold standard i.e. biopsy, is imperative to reduce patient morbidity and definitely mortality. Further studies dealing with the biological behavior of these non-malignant entities should be encouraged for better understanding of disease and disease progression so as to make necessary amendments in the treatment protocols.

\section{References}

[1]. Sandhya PG, Madhusudan C, Naseem N, Balakrishnan CD, Balagurunathan K. Interpretation of Upper GastroIntestinal Tract Endoscopic Mucosal Biopsies - A Study Conducted In Teaching Hospital In Puducherry, India. International Journal of Medical and Health Sciences, 2012; 1(3): $17-24$.

[2]. Rosai J: In Rosai and Ackerman's surgical pathology. 9th ed. St. Louis: Mosby; 2004. pp648-11.

[3]. Gill MK, Jain K, Manjari M, Kaur T. Expression of Her-2/neu in Colon Carcinoma and Its Correlation with the Histological Grades and the Lymph nodes status. Journal of Clinical and Diagnostic Research.2011 December, Vol-5(8): 1564-68.

[4]. Scheull B, Gruenberger T, Scheithauer W, Zielinski Ch, Wrba F. Her 2/neu protein expression in colorectal cancer. BMC Cancer 2006,6:123-27.

[5]. Sulegaon R, Shete S, Kulkarni D. Histological Spectrum of Large Intestinal Lesions with Clinicopathological Correlation. J Clin Diagn Res. 2015;9(11): EC30-EC34.

[6]. Shennak MM, Tarawneh MS, Al-Sheik; Upper gastrointestinal diseases in symptomatic Jordanians: A prospective endoscopic study. Annals of Saudi Medicine, 1997; 17(4): 471-474.

[7]. Hassan Abdulla Al-aquili. Clinical, colonoscopic and histopathological evaluation of lower gastrointestinal bleeding. Kufa Med, Journal 2012; Vol 15.

[8]. Tony J, Harish K, Ramachandran TM, Sunilkumar K, Thomas V. Profile of colonic polyps in a southern Indian population. Indian J Gastroenterol 2007;26:127-9.

[9]. Ullman T, Odze R, Farraye FA. Diagnosis and Management of Dysplasia in patients with Ulcerative Colitis and Crohn's Disease of the Colon. Inflamm Bowel Dis. 2009 April; 15(4):360-68.

[10]. Memon F, Baloch K, Memon AA; Upper gastrointestinal endoscopic biopsy; morphological spectrum of lesions. Professional Medical Journal, 2015; 22(12):1574-1579.

[11]. Neil A Shepherd, Roland M Valori; Guidance for Endoscopic Biopsy in the Gastrointestinal Tract Frontline. Gastroenterology, 2014; 5(2): 84-87 